\title{
Unanticipated Difficulty in an Anticipated Difficult Airway in the Neurointervention Suite: A Case Report
}

\author{
Rajashree Uday Gandhe ${ }^{1} \quad$ Chinmaya Pradeep Bhave ${ }^{1}$ \\ ${ }^{1}$ Division of Anaesthesiology, Department of Neuroanaesthesia, \\ Kokilaben Dhirubhai Ambani Hospital, Mumbai, Maharashtra, India
}

\author{
Avinash Sahebarav Kakde ${ }^{1} \quad$ Kalyani Anand Sathe ${ }^{1}$
}

J Neuroanaesthesiol Crit Care 2018;5:190-192

\begin{abstract}
Address for correspondence Rajashree Uday Gandhe, MD, Division of Anaesthesiology, Department of Neuroanaesthesia, Kokilaben Dhirubhai Ambani Hospital, 101 Simran, 15th Road, Khar Road, Mumbai 52, Maharashtra, India (e-mail: rajashreeug@gmail.com).
\end{abstract}

\author{
Abstract \\ Keywords \\ - difficult airway \\ - neurointervention \\ - vascular malformation
}

\begin{abstract}
Airway management of patients with craniofacial vascular malformations poses many challenges. Establishment of a secure airway is a prerequisite for safe anesthetic management of these patients. We report a case of a 45 -year-old man presenting with a facial vascular malformation involving the tongue, parapharynx, and extending into the neck, resulting in airway compromise scheduled for endovascular embolization.
\end{abstract}

\section{Introduction}

Airway assessment and management is an important domain of the anesthesiologist. Securing the airway with an endotracheal tube is often required for adequate airway control. In anticipated difficult airway situations where conventional direct laryngoscopy is anticipated to be difficult, fiberoptic bronchoscope-guided awake endotracheal intubation has gained importance. However, upper airway obstruction (near complete) is a relative contraindication to fiberoptic intubation. ${ }^{1}$ In such cases, elective tracheostomy under local anesthesia is the only possible alternative for securing the airway.

Venous malformations are soft tissue masses arising from the congenital disruption of normal venous development. They frequently appear in the head and neck region. Venous malformations are the third most vascular mass following hemangiomas and lymphatic malformations. ${ }^{2}$ Treatment options include surgical excision, laser ablation, cryosurgery, or endovascular sclerotherapy using steroids and sclerosing agents such as sodium tetradecyl sulfate. ${ }^{3}$

In this report, we describe the airway management done in the neurointervention suite for a 45-year-old man with large right facial vascular malformation compromising the airway.

\section{Case Report}

A 45-year-old man, with American Society of Anesthesiologists (ASA) physical status I, presented with progressive breathing difficulty since few months (-Fig. 1). The patient felt breathless more in the supine position and was comfortable in the sitting position. His vitals and systemic examination were unremarkable. On preoperative examination, a swelling over the right side of the face extending into the neck anteriorly was seen. Airway examination showed a large hypervascular growth occupying the whole oral cavity making further visualization of oropharyngeal structures

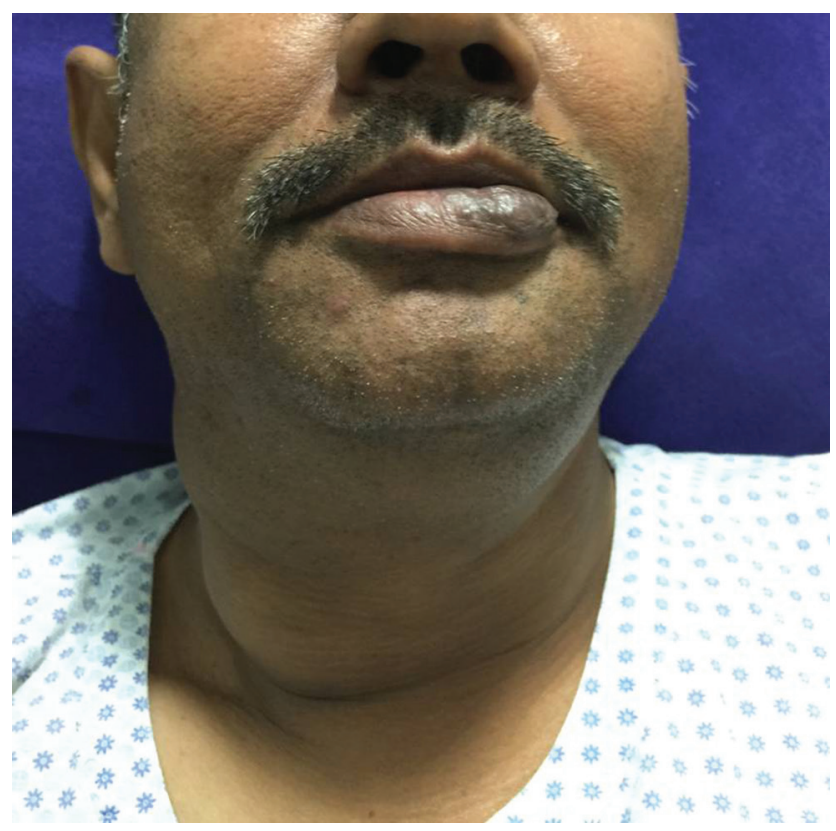

Fig. 1 Photograph of the patient showing the swelling over right side of the face and neck.

\section{received}

February 27, 2018 accepted after revision

April 27, 2018

published online

May 11, 2018
DOI https://doi.org/

10.1055/s-0038-1655899.

ISSN 2348-0548.
Copyright $\odot 2018$ Indian Society of Neuroanaesthesiology and Critical Care
License terms

(요 (1) $\odot$ 
difficult. Awake video laryngoscopy done previously showed extensive hypervascular growth involving right base of the tongue, valleculae, epiglottis, pyriform fossa, and bilateral false and true vocal cords. Findings of computed tomography $(\mathrm{CT})$ of the neck were diffuse swelling on right side of the neck extending superiorly into the right parapharyngeal space; inferiorly into the thyroid cartilage; medially into the right aryepiglottic fold, false vocal cord; anteriorly into base of tongue; and posteriorly into the anterior to carotid space (-Fig. 2). Magnetic resonance imaging (MRI) confirmed CT findings (-Figs. 3,4 ). The patient was thus scheduled for embolization of the vascular malformation. Based on the clinical features and radiologic findings, the decision was taken to go ahead with awake elective tracheostomy for securing the airway. Preoperative routine investigations such as complete blood count, serum creatinine, and coagulation

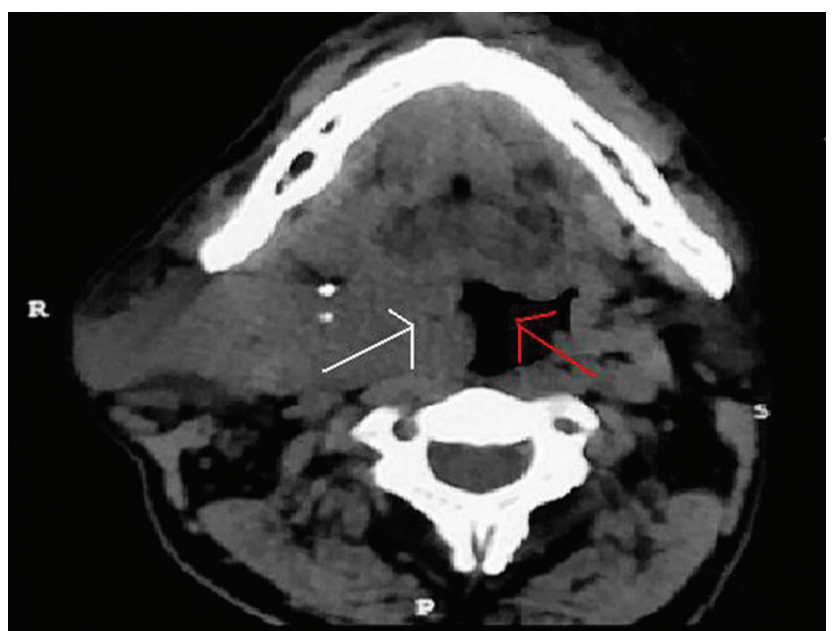

Fig. 2 Computed tomography scan of the neck showing relation of venous malformation (white arrow) with airway (red arrow).

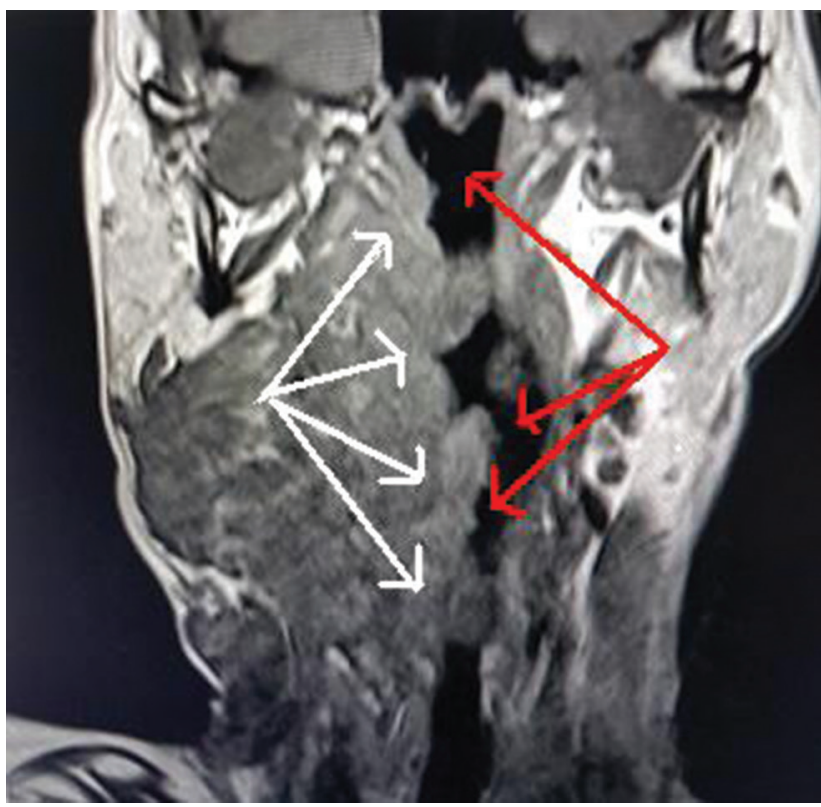

Fig. 3 Magnetic resonance imaging scan of the neck showing relation of venous malformation (white arrows) with airway (red arrows). profile were normal. Adequate blood was reserved for the procedure. Written informed high-risk consent was obtained from the patient. The patient was kept nil orally overnight.

On the day of procedure as per plan, neuroanesthesia team, ENT (ear-nose-throat), neurointervention, and critical care teams were present for the procedure in the neurointervention suite. The patient was wheeled in. All ASA standard monitors were attached. Two large-bore intravenous accesses were secured. No sedative premedication was administered to the patient. The patient was oxygenated with $100 \%$ oxygen. Because of the vascular malformation, surgical landmarks were difficult to identify. Awake surgical tracheostomy was attempted under local anesthesia. At incision, uncontrolled massive bleeding occurred. Attempt to control bleeding with pressure application was unsuccessful, and further dissection was abandoned but meanwhile the patient started desaturating. Saturation improved with bag-mask ventilation with $100 \%$ oxygen and intermittent emergency oxygen flush. Bleeding continued incessantly irrespective of all the measures taken by the operative surgeon. Moreover, aspiration of blood into the lower respiratory tract caused further deterioration of the patient's condition such as bradycardia, hypotension, and desaturation. Percutaneous tracheostomy was challenging in this patient as the neck anatomy was distorted and there was an increased risk of further blood loss and desaturation due to aspiration of blood into the trachea. As a lifesaving measure, percutaneous tracheostomy was attempted, and with great difficulty, it was achieved. Tracheostomy tube (TT) position was confirmed with capnography and fluoroscopy. Blood loss was approximately $1,000 \mathrm{~mL}$, and the patient was resuscitated with colloid and blood transfusion.

Digital subtraction angiography was done after stabilization of the patients' vital parameters. It did not suggest of

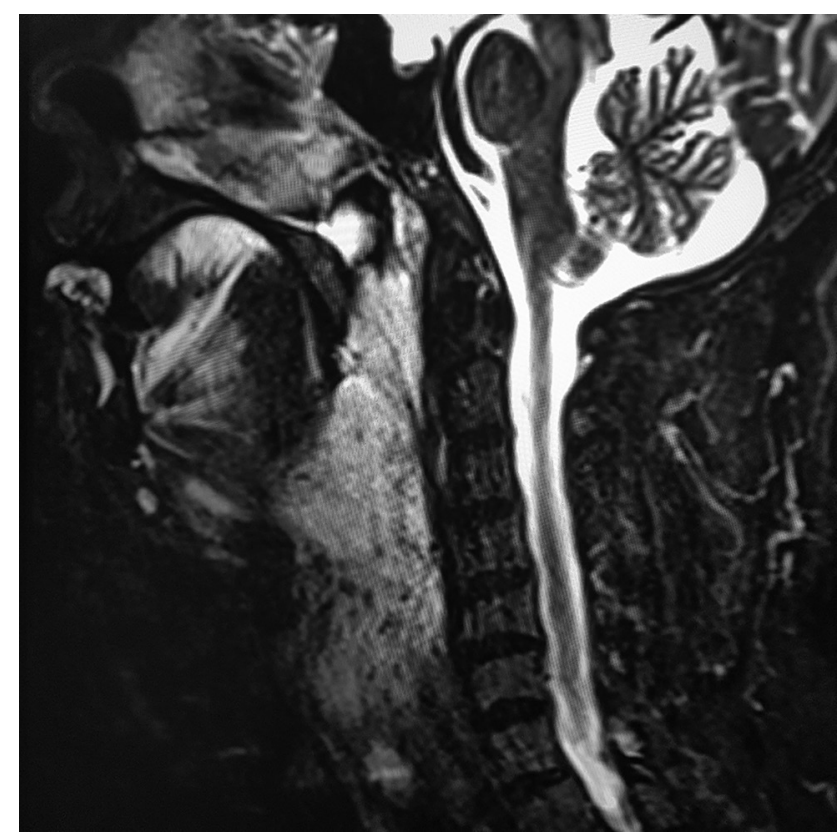

Fig. 4 Magnetic resonance imaging scan sagittal view showing venous malformation posterior to the tongue and anterior to the cervical spine. 
any arterial component to the parapharyngeal venous malformation. Sclerotherapy with injection of sodium tetradecyl sulfate was achieved. The patient was shifted to the intensive care unit (ICU) with TT in situ, where he developed systemic inflammatory response syndrome leading to acute respiratory distress syndrome and stress-induced cardiomyopathy.

The patient was managed in ICU successfully. His condition improved gradually, and he was shifted to the ward after 2 weeks. After rehabilitation and speech therapy, he was discharged with TT in situ. Complete closure of venous malformation was achieved with subsequent sclerotherapy treatment.

\section{Discussion}

Venous malformations are composed of anomalous veins with ill-defined boundaries that infiltrate normal tissue. They frequently appear in the head and neck region. If left untreated, venous malformations continuously expand and develop thrombi, become painful, and disrupt normal function. ${ }^{2}$

Anesthetic management of vascular malformations depends on location, extent, age, and clinical presentation. Additional anesthetic considerations in facial venous malformations are difficult intubation, airway compression, and obstructive sleep apnea. ${ }^{4}$ In patients with vascular soft tissue mass, imaging confirms clinical history and it is diagnostic. MRI helps in determining extent of venous malformation, evaluation of adjacent neurovascular structure, and inflow and outflow channels. Treatment options include surgical excision (laser or cryosurgery), sclerotherapy, and embolization.

Neurointervention suite is a place where handling difficult airway and related emergencies add to the challenges of anesthetic management. Training of the personnel is also not adequate to handle difficult airway emergencies compared with operating theater. This presents an additional challenge for the attending anesthesiologist.

In our case, venous malformation involved almost the entire pharynx and larynx. Increased vascularity and friability of the lesion added to the risks. Any fiddling with it during airway management could have caused uncontrolled bleeding from lesion. Hence awake fiberoptic that is normally considered as a standard technique of securing difficult airway was not possible here. Therefore, elective awake tracheostomy was planned.
Surgical tracheostomy is believed to be the safest option in difficult airway situations. However, in our case we faced unanticipitated complications such as bleeding and airway compromise. Several factors influence choice of technique to manage the emergency airway, which includes anatomical, user experience, and available devices.

Percutaneous dilatational tracheostomy was performed successfully, and airway was secured. Although percutaneous tracheostomy is not advisable in emergency situations, it can be safely performed under experienced hands. It may be easier and faster than surgical tracheostomy. ${ }^{5}$

\section{Conclusion}

Patients with vascular lesions in the head and neck region have potential to cause problems in airway management and massive bleeding. Preoperative clinical assessment and radiologic findings are crucial to diagnose potential airway difficulties. Surgical tracheostomy is considered to be the final option in difficult airway management, but catastrophic complications may arise during the procedure. Current knowledge of available airway management alternatives would enable us to overcome difficult airway difficulties.

\section{Conflict of Interest}

None.

\section{References}

1 Joshi C, Ambi U, Prakashappa DS, Mirji P. Anaesthetic considerations in macroglossia due to haemangioma of the tongue: a case report. J Clin Diagn Res 2011;5:840-842

2 Richter GT, Braswell L. Management of venous malformations. Facial Plast Surg 2012;28(6):603-610

3 Bajwa SS, Panda A, Bajwa SK, Singh A, Parmar SS, Singh K. Anesthetic and airway management of a child with a large upper-lip hemangioma. Saudi J Anaesth 2011;5(1):82-84

4 Griffith CL, Herod J. Anaesthesia for massive venous malformation with DIC and strategies for minimising blood loss. Case report. Pediatr Anesthesia Crit Care J 2015;3:22-25

5 Schmitz S, Boven MV, Hamoir M. Percutaneous tracheostomy in emergency situation setting. Ann Otolaryngol Rhinol 2015;2:1028 